\title{
Anomalous EDGES 21-cm signal and a moduli dominated era
}

\author{
Mansi Dhuria* \\ Institute of Infrastructure Technology Research and Management, Ahmedabad 380026, India
}

(Received 8 June 2019; published 13 November 2019)

\begin{abstract}
The EDGES Collaboration has recently reported the detection of an unexpectedly stronger absorption signal in the global $21-\mathrm{cm}$ spectrum around cosmic redshift $z=17$, resulting in significant cooling of the primordial gas. The cooling of the gas can be expected to occur by considering the scattering off baryons by a small fraction of dark matter (DM) carrying a tiny electromagnetic charge (millicharged DM). Prior works have shown that the relic density of millicharged DM obtained by using the standard thermal freeze-out mechanism gets overproduced in the range of parameters allowed by EDGES 21-cm signal. Thus, millicharged DM will not be able to explain the EDGES signal successfully unless one invokes new exotic particles or force carriers. In this study, we argue that this problem can be evaded if the postinflationary Universe undergoes a period of an early matter/modulus domination, which one generally encounters while working in the framework of UV complete theories. By taking this into account, we estimated the relic abundance of millicharged DM and found that it is possible to obtain the desired fraction of millicharged $\mathrm{DM}$ for a narrow range of requisite parameters by taking modulus mass around $10^{3} \mathrm{TeV}$. Quite interestingly, an almost similar value of modulus mass is required to obtain the right value of inflationary bounds and around $\mathrm{TeV}$-scale supersymmetry in one of the promising string compactification scenarios, dubbed as the large volume scenario.
\end{abstract}

DOI: $10.1103 /$ PhysRevD.100.103007

\section{INTRODUCTION}

In spite of the rigorous search over decades, the nature of dark matter (DM) still remains very puzzling. Although almost all of our evidence for the existence of DM mainly relies on its gravitational interactions with an ordinary baryonic matter, we expect that it should have some level of nongravitational interaction with standard model (SM) baryons. In fact, there exist a few puzzles at a small scale of the Universe which require DM to have nonzero gravitational interactions $[1,2]$. The attempts to probe the exact nature of DM are being carried out intensively in a wide range of direct and indirect detection experiments, accelerator-based searches, and some of the cosmological observations. However, unfortunately, none of these experiments has been able to find a conclusive evidence for the nongravitational interactions of DM till now.

An interesting novel probe to search for nongravitational interactions of DM with ordinary baryonic gas relies on studying the redshifted $21-\mathrm{cm}$ signal of neutral hydrogen in the cosmic dawn. The first detection of the $21-\mathrm{cm}$ signal

\footnotetext{
*mansidhuria@iitram.ac.in
}

Published by the American Physical Society under the terms of the Creative Commons Attribution 4.0 International license. Further distribution of this work must maintain attribution to the author(s) and the published article's title, journal citation, and DOI. Funded by SCOAP . from the cosmic dawn has been reported recently by an Experiment to Detect the Global Epoch of reionization Signature (EDGES) [3]. After removing the instrumental noise and foregrounds, the extracted signal features a broad absorption dip with brightness temperature $T_{21}=$ $-500_{-500}^{+200} \mathrm{mK}$, centered at redshift $z=17.2$. In the standard cosmological model, the maximum possible brightness temperature expected at $z=17$ is around $209 \mathrm{mK}$. This value corresponds to a baryon gas temperature $T_{b} \sim 7 \mathrm{~K}$. However, the observed $T_{21}=-500 \mathrm{mK}$ requires the primordial gas to be much colder, $T_{b} \sim 4 \mathrm{~K}$, which is difficult to explain by astrophysical models alone [3]. It has been argued in Refs. [4-6] that the observed discrepancy can be accounted for by considering the scattering off the baryons by light DM, thus draining excess energy from the primordial gas. The significant cooling at a particular redshift can follow from the elastic scattering between DM and baryons with a Rutherford cross section with $1 / v^{4}$ dependence, where $v$ is the relative velocity between DM and baryons $[7,8]$. In the context of particle physics models, the $v^{-4}$ cross section corresponds to DM interacting with baryons either through a massless photon or a light mediator with mass smaller than the momentum transfer. However, tight constraints from the fifth force experiments and limits from stellar cooling completely rule out the possibility of light mediators [9]. Several studies have explored the millicharged DM interpretation of the EDGES signal by considering its 
annihilation through SM photons [10-12] and an additional vector boson $[9,11,13,14]$. For the case of millicharged DM interacting through SM photons, it has been found in Refs. $[10,11]$ that the viable parameter space of the $21-\mathrm{cm}$ signal can be obtained only if a small fraction of DM $\left(f_{\mathrm{DM}} \approx 0.02\right)$ is charged. However, the relic density of the millicharged DM obtained through standard thermal freezeout gets overproduced in the allowed region of parameter space. Thus, the whole parameter space required to explain the $21-\mathrm{cm}$ signal gets ruled out from the requirement of obtaining the desired DM fraction. In case of additional massive gauge bosons, all the parameter space gets ruled out mostly from fifth-force constraints [9].

In this work, we explore the possibility of depletion of an overproduced relic density of millicharged DM by taking into account the presence of an early matter/moduli dominated era. Moduli are the massless scalar fields, which appear generically in the consistent string compactifications [15-20]. Their presence generically gives rise to a period of a matter dominated era in the early Universe. Depending on the mass and charge of DM, it is also possible for millicharged DM to get decoupled in the matter dominated era. Since the expansion rates are different for both the matter and radiation dominated eras, this would certainly yield different values of the final relic abundance in both cases. The mechanisms to produce DM in moduli dominated cosmology have already been discussed in the literature; see, e.g., Refs. [19-23].

In this work, we show that both the desired fraction of DM relic density and an absorption signal of $21 \mathrm{~cm}$ can be explained simultaneously, albeit for a narrow range of mass and charge of DM by taking into account the modulus dominated era. Quite interestingly, this also sets a bound on the mass of modulus $m_{\Phi} \approx 10^{3} \mathrm{TeV}$, which almost matches with the value of modulus mass given in one of the promising string compactification scenarios, dubbed the large volume scenario [24-27] in order to obtain the observed inflationary predictions and $\mathcal{O}(\mathrm{TeV})$-scale supersymmetry breaking [28].

The paper is structured as follows. In Sec. II, we discuss constraints obtained on the mass and charge of millicharged $\mathrm{DM}$ from the measurements of the $21-\mathrm{cm}$ line signal reported by the EDGES Collaboration, along with several experimental and astrophysical constraints. In Sec. III, we briefly discuss the dynamics of the moduli present in the early Universe and the possibility of millicharged DM getting decoupled in an early modulus/matter dominated era. In Sec. III A, we present the detailed derivation of calculating relic abundance of DM in the matter dominated era. In Sec. III B, we give various expressions useful to calculate the amount of dilution in the energy density of DM particles after the decay of modulus. In Sec. IV, we apply our results of the relic abundance obtained during matter domination to a case with millicharged DM. In Sec. IV A, we give constraints on the mass of the modulus.
In Sec. IV B, we show different regions in the parameter space of mass and charge of millicharged DM allowed by the EDGES 21-cm line signal and a particular fraction of millicharged DM relic density in both the matter and radiation dominated eras. The results show that, unlike the radiation dominated era, it is possible to obtain the desirable fraction of millicharged DM relic density in the range of parameters allowed by $21-\mathrm{cm}$ line signal if DM gets decoupled in an early modulus dominated era. In Sec. V, we summarize our results.

\section{MILLICHARGED DM AND 21-CM LINE SIGNAL}

It was discussed in Ref. [10] that the required $21-\mathrm{cm}$ absorption features can be explained if the millicharged DM forms only a small fraction of the entire DM of the Universe. After solving the Boltzmann equation for the temperature of baryon gas (by taking into effect the interaction of DM with baryons), the authors of Ref. [10] found out that the baryon temperature $T_{b} \approx 4 \mathrm{~K}$ can be obtained if the charge of DM satisfies the following constraint,

$$
\epsilon \approx 6 \times 10^{-7}\left(\frac{m_{X}}{\mathrm{MeV}}\right)\left(\frac{f_{\mathrm{DM}}}{10^{-2}}\right)^{-3 / 4}
$$

where $f_{\mathrm{DM}}$ is the fraction of the DM possessed by millicharged particles. In addition to the constraint on the charge of DM, in order for DM to cool the baryonic gas efficiently, the equipartition theorem requires that the mass of DM particles should be $m_{X}<6.2 \mathrm{GeV} \times f_{\mathrm{DM}}$.

Now, the parameters of millicharged DM are also subjected to the constraints from various different experiments. Following Refs. [10,11], we take into account the constraints from observations of supernovae SN1987A [29]; the SLAC millicharge experiment [30]; bounds on the abundances of light elements produced during big bang nucleosynthesis (BBN), i.e., $\Delta N_{\text {eff }}$ [31]; constraints from measurements of the cosmic microwave background (CMB) on DM annihilation in the epoch of recombination [32]; and DM scattering with baryons [33]. The constraints on $\Delta N_{\text {eff }}$ depend on which SM particle it couples to and the nature of DM particle [34]. The constraints on dark matter scattering with baryons during kinetic decoupling (KD) in Ref. [33] and DM annihilation in the epoch of recombination in Ref. [32] are presented for $f_{\mathrm{DM}}=1$. Based on the analytical expressions given in Refs. [32,33], we have rescaled the constraints on DM annihilation constraints by a factor of $f_{\mathrm{DM}}^{2}$ and the DM scattering cross section by a factor of $\sqrt{f_{\mathrm{DM}}}$. However, one should note that the scaling argument only results in approximate constraints on a particular fraction of DM relic abundance $\left(f_{\mathrm{DM}} \Omega_{\mathrm{DM}}\right)$ in both cases. The constraints from DM scattering with baryons would not apply for the case of $f_{\mathrm{DM}} \lesssim 0.01$ because the energy density of the millicharged DM is less than the difference between the upper limit (at 95\% C.L.) 
imposed on the baryon density from $\mathrm{CMB}$ and the lower limit (at 95\% C.L.) imposed on the baryonic density from BBN [11].

All of the aforementioned constraints are shown in the form of different shaded regions in Fig. 1. In this figure, we have also shown the region in the parameter space required to explain the $21-\mathrm{cm}$ absorption feature as reported by the
EDGES Collaboration, along with the parameter space allowed from the requirement of obtaining the desired fraction of millicharged DM abundance in both the matter and radiation dominated eras. Our results in Fig. 1 would clearly point out that that the issue related to the overproduction of DM density [11] in the desirable range of the mass and charge of DM can be avoided if DM gets

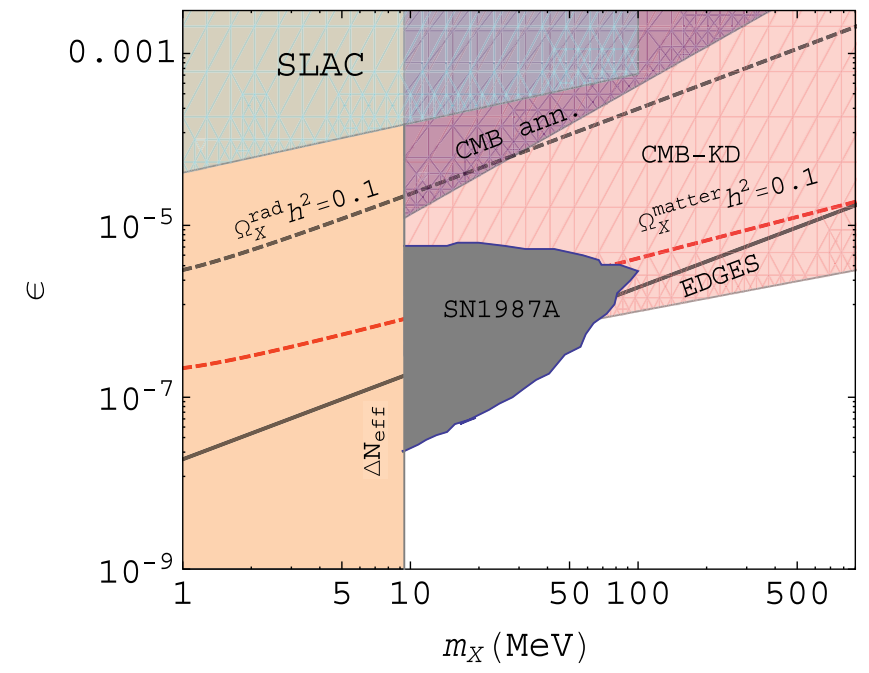

(a) Millicharged DM fraction $f_{\mathrm{DM}}=1$

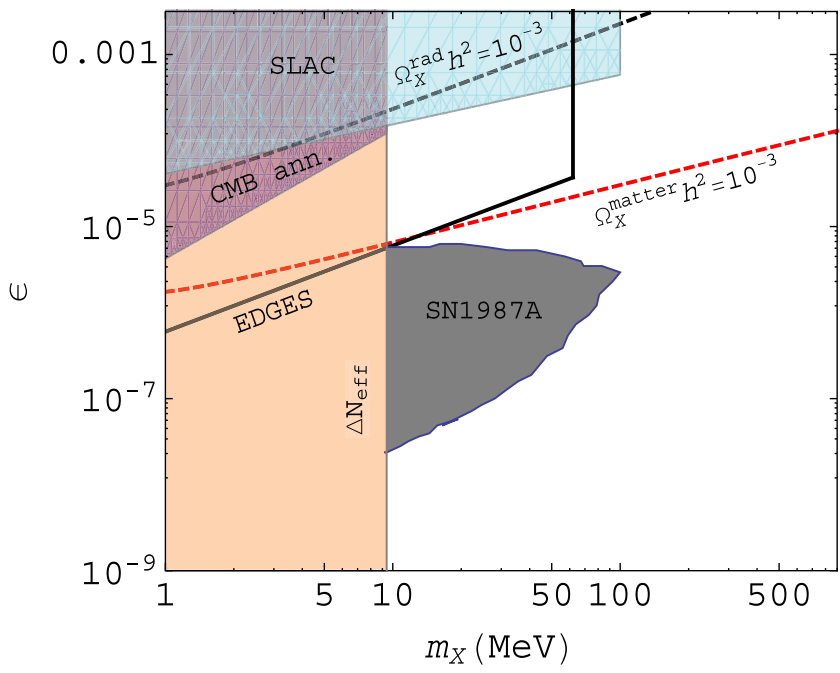

(c) Millicharged DM fraction $f_{\mathrm{DM}}=0.01$

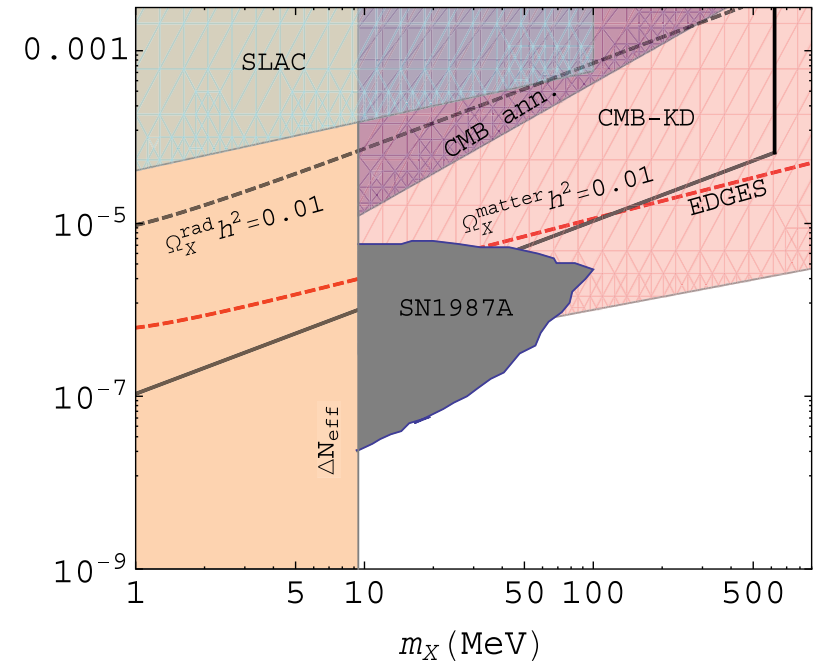

(b) Millicharged DM fraction $f_{\mathrm{DM}}=0.1$

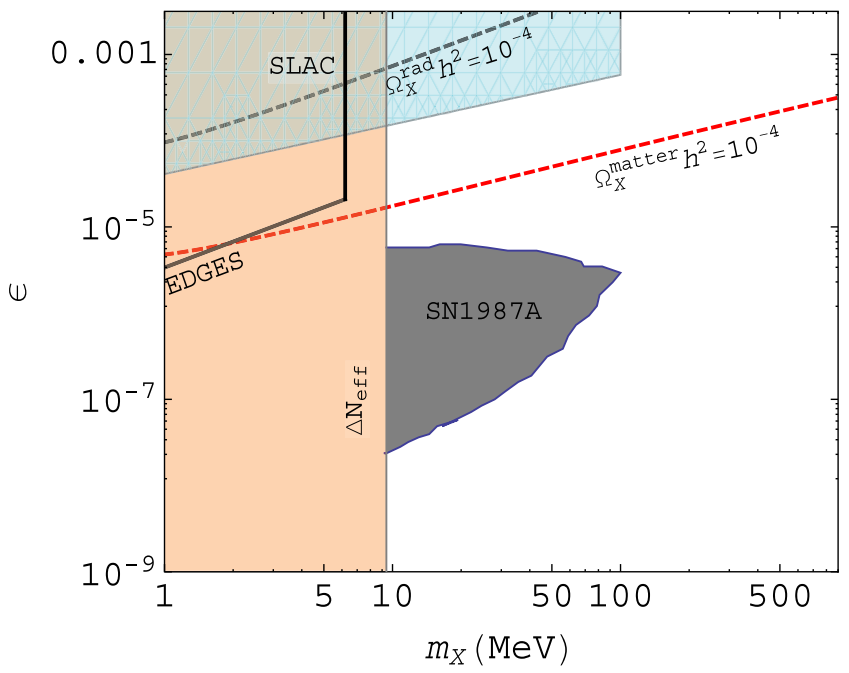

(d) Millicharged DM fraction $f_{\mathrm{DM}}=0.001$

FIG. 1. We show the region in the parameter space for which the desirable value of relic abundance can be obtained by considering an early modulus dominated era. The black solid lines in Figs. 1(a)-(d) indicate the ranges of DM parameters required to explain the observed 21-cm signal reported by the EDGES Collaboration. The dashed red line in each figure indicates the parameter space for which the relic abundance of millicharged DM abundance becomes equal to the quoted fraction $f_{\mathrm{DM}}$. The black dashed lines in Figs. 1(a)-(d) indicate the parameter space required to get a particular fraction of millicharged DM if the same gets decoupled in the radiation dominated era. We also show constraints from Supernova 1987A (dark gray, labeled SN 1987A), the SLAC millicharge experiment (cyan, labeled SLAC), production of light element abundances produced during BBN (light orange, labeled $\Delta N_{\text {eff }}$ ), and approximate constraints from DM scattering with baryons (pink, labeled CMB-KD) and DM annihilation (dark purple, labeled CMB ann). One can see that the dashed black lines do not coincide with solid black lines, while the dashed red line intersects with the solid black line for a particular value of mass and charge of DM in each figure. Thus, the millicharged DM can consistently explain the 21-cm EDGES signal if it is assumed to be decoupled in an early modulus dominated era. 
produced in an early matter dominated era. Before we discuss our results in Sec. IV, we will first derive the expression to calculate the relic abundance of DM in the matter/modulus dominated era. The readers not interested in going through steps of the derivation of DM relic abundance can directly use the final expression of DM relic abundance in Eq. (23) and then move to the final results in Sec. IV.

\section{MODULI DOMINATED COSMOLOGY}

Moduli are the massless scalar fields mostly appearing in string compactifications. Because of their gravitational interactions, they get significantly displaced from the minimum of the potential just after the end of inflation [15-20]. Subsequently, the energy density associated with moduli fields begins to redshift like matter. Since it scales as $1 / a^{3}$ during the expansion of the Universe (in comparison to the energy density of radiation being scaled as $1 / a^{4}$ ), they start dominating the Universe quickly after the end of inflation, leading to an early period of a matter dominated era. Eventually, when the Hubble expansion rate becomes of the order of $\Gamma_{\text {mod }}$, moduli decay, and the Universe enters the radiation dominated era. The decay of moduli into relativistic SM particles would increase the entropy of the Universe, thus again reheating the Universe. Since one requires that the decay of moduli into SM particles shall not disturb the constraints imposed on the abundances of light elements produced by BBN, one has to assume that either decay happens before the onset of BBN (i.e., $T \geq \mathrm{MeV}$ ) or the nal modulus reheating temperature is greater than the BBN temperature. For the decay width of a modulus given by $\Gamma_{\Phi} \sim m_{\Phi}^{3} / M_{p}^{2}$, the reheating temperature $T_{r}$ (roughly given by $\left.\sqrt{\Gamma_{\Phi} M_{p}}\right)$ will be greater than $\mathcal{O}(\mathrm{MeV})$ if its mass is greater than $\mathcal{O}(\mathrm{TeV})$ [15-19].

In the standard cosmology, we assume that the Universe is dominated by radiation from the point of inflationary reheating until the period of BBN. Therefore, DM also gets decoupled during radiation domination. Since the inclusion of the modulus allows an early period of matter domination, it is not necessary for the DM decoupling to occur during radiation domination. We consider a scenario in which the early Universe is formed of a thermal bath, comprising the visible sector states, dark matter, and a single moduli field (dubbed as the modulus) [35]. Depending on the freeze-out temperature of $\mathrm{DM}\left(T_{\mathrm{FO}}\right)$, modulus decay temperature $\left(T_{\Gamma}\right)$, and the temperature at which the modulus started dominating $\left(T_{*}\right)$, three distinct regimes might occur [36]:

(1) Freeze-out after moduli domination.- - If $T_{\Gamma}>T_{\mathrm{FO}}$, DM particles will get decoupled in the radiation dominated era. This is the standard radiation domination case. Thus, the conventional thermal freeze-out would work. As it has already been discussed in Refs. [10,11] that the relic density of DM will always be overproduced for the values of
DM parameters required to explain the $21-\mathrm{cm}$ absorption feature for conventional thermal freezeout case, this case is already ruled out.

(2) Freeze-out before moduli domination.-If $T_{\mathrm{FO}}>$ $T_{*}, T_{\Gamma}$, DM particles would again decouple during the radiation dominated era. However, since the modulus starts dominating at $T_{*}<T_{\mathrm{FO}}$ and then decays at $T_{\Gamma}$, the overall relic density of already decoupled DM particles will get diluted because of an overall increase in the entropy of the Universe after the decay of the modulus. For the case of millicharged DM with mass approximately $\mathrm{GeV}$, freeze-out temperature $T_{\mathrm{FO}}<\mathrm{GeV}$. On the other hand, for $m_{\Phi} \geq 10 \mathrm{TeV}$ (constrained by BBN) and $T_{*} \sim \sqrt{m_{\Phi} M_{p}} \geq 5 \times 10^{6} \mathrm{TeV}$ [see Eq. (17)], one would typically have $T_{\mathrm{FO}} \ll T_{*}$. Thus, it is unlikely that millicharged DM will fall in this regime.

(3) Freeze-out during moduli domination.-If $T_{*}<$ $T_{\mathrm{FO}}<T_{\Gamma}$, DM particles will get decoupled in the matter dominated era. Since the expansion rate in the matter domination case scales differently as compared to the radiation dominated case, the presentday relic abundance of DM is expected to be different than the standard radiation domination case. Since it is possible to have $T_{*}<T_{\mathrm{FO}}<T_{\Gamma}$, one needs to see the effect of an early matter domination in the final relic abundance of DM.

In the next subsection, we calculate the relic abundance of millicharged DM particles by taking the aforementioned case of matter domination.

\section{A. Relic abundance of DM in matter dominated era}

The number density $n_{X}$ of DM particles will follow the standard form of the Boltzmann equation given by

$$
\dot{n}_{X}+3 H n_{X}=-\langle\sigma v\rangle\left[n_{X}^{2}-\left(n_{X}^{\mathrm{eq}}\right)^{2}\right],
$$

where $\langle\sigma v\rangle$ is the thermally averaged cross section of DM particles. By using $H=\dot{T} / T$, we can rewrite the lhs in terms of the comoving number density given by $Y=n_{X} / s$. Defining $x=m_{X} / T$ and $\langle\sigma v\rangle=\sigma_{0} / x^{n}$, we will have

$$
H x^{n+1} \frac{d Y}{d x}=-\sigma_{0} s\left[Y_{X}^{2}-\left(Y_{X}^{\mathrm{eq}}\right)^{2}\right],
$$

where $s=\frac{2 \pi^{2}}{45} g_{*} T^{3}$ is the entropy density. The expansion rate $H$ is generally given by

$$
H^{2}=\frac{8 \pi}{3 M_{p}^{2}}\left(\rho_{\gamma}+\rho_{\Phi}+\rho_{\mathrm{DM}}\right) .
$$

We can parametrize $H=H(T)$ in terms of critical temperature $T_{*}\left(T_{*}>T>m_{X}\right)$, and the relative fraction of the 
$\mathrm{DM}$ and SM number density given by $r=\frac{\rho_{\gamma}+\rho_{\mathrm{DM}}}{\rho_{\gamma}+\rho_{\Phi}+\rho_{\mathrm{DM}}}$ as follows [36],

$$
\frac{H^{2}}{H_{*}^{2}}=r\left(\frac{a_{*}}{a}\right)^{4}+(1-r)\left(\frac{a_{*}}{a}\right)^{3},
$$

where $H_{*}=H\left(T_{*}\right), a_{*}=a\left(T_{*}\right)$, and $T_{*}$ corresponds to the temperature after which the matter domination era begins to start. Since thermal plasma is formed of radiation, by rewriting $a_{*} / a$ in terms of $T / T_{*}$, the Hubble expansion rate will be [36]

$H=H_{*}\left(\frac{g_{*}\left(T_{*}\right)}{g_{*}(T)}\right)^{3 / 8}\left(\frac{T}{T_{*}}\right)^{3 / 2}\left[(1-r)+r\left(\frac{T}{T_{*}}\right)\right]^{1 / 2}$.

Note that for the radiation dominated case, i.e., $r \approx 1$, we reproduce the standard form of $H \propto T^{2}$, whereas for the modulus/matter domination case, i.e., $r \ll 1$, it gives $H \propto T^{3 / 2}$. By rewriting $Y$ as $\Delta=Y-Y_{\text {eq }}, x=m / T$ and using the expression of $H$ given in Eq. (6), we can write

$\Delta^{\prime} \approx-Y_{\mathrm{eq}}^{\prime}-\lambda\left(1-r+r \frac{x_{*}}{x}\right)^{-1 / 2} x^{-5 / 2-n} \Delta\left[2 Y_{\mathrm{eq}}+\Delta\right]$,

where primed variables correspond to derivatives with respect to $x$ and $\lambda=2 \pi^{2} g_{* S} m_{X}^{3} \sigma_{0} /\left(45 H_{*} x_{*}^{3 / 2}\right)$. Since we are interested in obtaining $Y$ for $x>x_{\mathrm{FO}}$, we will always have $Y>Y_{\text {eq. }}$. Thus, we can write the late time difference $\Delta \sim Y$ and may also ignore $Y_{\text {eq }}^{\prime}$ in Eq. (7). With this, the present time $\Delta_{\infty}=\left.\Delta\right|_{x>x_{\mathrm{FO}}}$ can follow from

$$
\Delta_{\infty}^{\prime} \approx-\lambda\left(1-r+r \frac{x_{*}}{x}\right)^{-1 / 2} x^{-5 / 2-n} \Delta_{\infty}^{2}
$$

By integrating the above equation from $x=x_{\mathrm{FO}}$ to $x \approx 0$, the freeze-out comoving number density will be

$$
Y_{\mathrm{FO}} \approx\left(\lambda\left(1-r+r \frac{x_{*}}{x}\right)^{-1 / 2} x^{-5 / 2-n}\right)^{-1} .
$$

By evaluating the integral for $r \ll 1$, we would obtain the following freeze-out comoving DM number density in the matter dominated case [36],

$Y_{\mathrm{FO}}^{\mathrm{matter}} \approx\left(n+\frac{3}{2}\right) \frac{x_{\mathrm{FO}}^{n+\frac{3}{2}}}{\lambda}=3 \frac{\sqrt{5}}{\pi} \frac{\sqrt{g_{*}}}{g_{* S}} \frac{\left(n+\frac{3}{2}\right) x_{\mathrm{FO}}^{n+\frac{3}{2}}}{M_{p} m_{X} \sigma_{0} \sqrt{x_{*}}}$,

while in the case of radiation domination, i.e., the $r \rightarrow 1$ limit, $Y_{\mathrm{FO}}^{\mathrm{rad}}$ is proportional to $\left(x_{F}^{\mathrm{rad}}\right)^{n+1}$. Thus, we can clearly see that the dependence of $Y_{\mathrm{FO}}$ on $x_{\mathrm{FO}}$ is different for the matter and radiation dominated eras. Additionally, $Y_{\mathrm{FO}}$ in the matter dominated era depends on the additional parameter $x_{*}$, which further depends on the details of dynamics of the modulus in the early Universe.

\section{Freeze-out temperature}

For the number density of nonrelativistic DM particles given by $n_{X}=\frac{g_{X}}{(2 \pi)^{3 / 2}} m_{X}^{3} x^{-3 / 2} e^{-x}$, the freeze-out temperature can be found by equating $H\left(T_{\mathrm{FO}}\right)=n_{X}\langle\sigma v\rangle$. By using the expression of $H$ given in Eq. (6), it turns out to be [36]

$$
x_{\mathrm{FO}}=\ln \left[\frac{g_{X} m_{X}^{3} \sigma_{0}}{(2 \pi)^{3 / 2} x_{*}^{3 / 2} H_{*}}\left(\frac{x_{\mathrm{FO}}^{-1+2 n}}{(1-r) x_{\mathrm{FO}}^{4 n-1}+x_{*} r}\right)^{1 / 2}\right] \text {, }
$$

where $H_{*}=H_{*}\left(T_{*}\right)$ and $x_{*}=m / T_{*}$. Now, by taking $r \ll 1$ and $H_{*}=8 \pi^{2} g_{*} T_{*}^{4} / M_{p}^{2}$, it gives the following value of $x_{\mathrm{FO}}$ in the matter domination case for $n=0$ :

$$
x_{\mathrm{FO}}^{\text {matter }} \approx \ln \left[\frac{3}{4 \pi^{3}} \sqrt{\frac{5}{3}} \frac{g_{X}}{g_{*}} \frac{m_{X}^{3 / 2} M_{p} \sigma_{0}}{T_{*}}\right] .
$$

This expression will be useful while calculating the value of the final relic abundance of DM.

\section{B. Dilution of relic density}

As discussed in Sec. III, the decay of the modulus into relativistic particles might increase the entropy of the Universe at late times, thus again reheating the Universe [19]. Therefore, if DM gets decoupled before the decay of the modulus (the case we are considering in this work), the comoving number density of DM particles produced during the matter dominated era in our case might get diluted after the decay of the modulus. In that case, the final freeze-out comoving number density will be given by [19]

$\left.Y_{\mathrm{FO}}^{\text {matter }}\right|_{\text {after }}=\left.Y_{\mathrm{FO}}^{\text {matter }}\right|_{\text {before }}\left(\frac{s_{\text {after }}}{s_{\text {before }}}\right)=\frac{\left.Y_{\mathrm{FO}}^{\text {matter }}\right|_{\text {before }}}{\xi}$,

where $\xi$ is the dilution factor. The entropy densities are given as $s_{\text {before }}=g_{* S}\left(T_{\Gamma}\right) T_{\Gamma}^{3}$ and $s_{\text {after }}=g_{* S}\left(T_{r}\right) T_{r}^{3}$, where $T_{\Gamma}$ and $T_{r}$ correspond to the decay temperature of the modulus and reheat temperature of the Universe after the decay of the modulus.

The decay temperature can be found by taking [19]

$$
3 H_{\text {decay }}^{2}=\frac{m_{\Phi} Y_{\Phi} s_{\text {decay }}}{M_{p}^{2}}=\frac{m_{\Phi} Y_{\Phi}}{M_{p}^{2}} \frac{2 \pi^{2}}{45} g_{* S}\left(T_{d}\right) T_{\Gamma}^{3},
$$

where $Y_{\Phi}=n_{\Phi} / s$ is the comoving number density of the modulus. For $H_{d}=\Gamma_{\text {mod }}$, the decay temperature $T_{\Gamma}$ will be given as 


$$
T_{\Gamma}=\left(\frac{30}{\pi^{3}}\right)^{1 / 3}\left(\frac{\Gamma_{\text {mod }}^{2} M_{p}^{2}}{m_{\Phi} Y_{\Phi} g_{* S}\left(T_{\Gamma}\right)}\right)^{1 / 3}
$$

In this case, $Y_{\Phi}=n_{\Phi} / s$ will be given by

$$
Y_{\Phi}=\frac{1}{2} m_{\Phi} f_{\Phi}^{2} s^{-1}\left(T_{*}\right)
$$

where $f_{\Phi}$ is the oscillation length of the modulus, which will be typically of an $\mathcal{O}\left(M_{p}\right)$. Here, $T_{*}$ is the temperature at which the modulus starts oscillating. Its value can be calculated as follows:

$$
\begin{aligned}
3 H^{2} & =3\left(\frac{1}{2} m_{\Phi}\right)^{2} M_{p}^{2}=M_{p}^{-2}\left(\frac{\pi^{2}}{30}\right) g_{*} T_{*}^{4} \\
\rightarrow T_{*} & =\left(\frac{90}{4 \pi^{2} g_{*}}\right)^{1 / 4}\left(m_{\Phi}^{2} M_{p}^{2}\right)^{1 / 4} .
\end{aligned}
$$

The reheating temperature after the decay of the modulus can be calculated from

$$
\begin{aligned}
3 H^{2} & =\frac{4 \Gamma_{\Phi}^{2}}{3}=\frac{\pi^{2}}{30} g_{*}\left(T_{r}\right) \frac{T_{r}^{4}}{M_{p}^{2}} \\
\rightarrow T_{r} & =\left(\frac{40}{\pi^{2}}\right)^{1 / 4} g_{*}^{-1 / 4}\left(T_{r}\right) \sqrt{\Gamma_{\Phi} M_{p}}
\end{aligned}
$$

For the decay width of the modulus given by $\Gamma_{\Phi}=D_{\Phi} \frac{m_{\Phi}^{3}}{M_{p}^{2}}$, the value of the dilution factor will follow from

$$
\begin{aligned}
\xi & =\frac{s_{\text {after }}}{s_{\text {before }}} \approx \frac{g_{* S}\left(T_{r}\right)}{g_{* S}\left(T_{\Gamma}\right)}\left(\frac{T_{r}}{T_{\Gamma}}\right)^{3} \\
& =\frac{2}{15}\left(250 \pi^{2}\right)^{1 / 4} g_{*}^{1 / 4}\left(\frac{M_{p}}{D_{\Phi} m_{\Phi}}\right)^{1 / 2} Y_{\Phi},
\end{aligned}
$$

where $Y_{\Phi}$ can be calculated by using Eqs. (16) and (17).

\section{Present DM relic abundance in matter dominated era}

The present-day relic abundance of DM for the same getting decoupled in the matter dominated era is given by

$$
\Omega_{0, \mathrm{DM}}^{\mathrm{matter}}=\frac{1}{\xi} \times \frac{s_{0} m_{X} Y_{\mathrm{FO}}}{\rho_{c}}
$$

where $s_{0}=\frac{2 \pi^{2}}{45} g_{* S}\left(T_{0}\right) T_{0}^{3}$, with $T_{0}=2.34 \times 10^{-10} \mathrm{MeV}$, and $\rho_{c}=8.05 \times 10^{-35} \mathrm{MeV}^{4}$. The value of $T_{*}$ can be calculated by using Eq. (17). Using Eq. (10) and Eqs. (16)-(19), for $g_{*}=g_{* S} \approx 100$, the DM relic abundance will be parametrized by

$$
\Omega_{0, \mathrm{DM}}^{\text {matter }} \sim 0.1\left[\sqrt{\frac{20 \mathrm{MeV}}{m_{\mathrm{DM}}}}\left[\frac{10^{-18} \mathrm{MeV}^{-2}}{\langle\sigma v\rangle}\right]\left[\frac{m_{\Phi}}{10^{9} \mathrm{MeV}}\right]^{5 / 4} \times\left(1-\frac{1}{47} \log \left[\left(\frac{m_{X}}{20}\right)^{3 / 2}\left(\frac{\langle\sigma v\rangle}{10^{-18}}\right)\left(\frac{m_{\Phi}}{10^{9}}\right)^{1 / 4}\right]\right)^{(3 / 2)}\right]
$$

\section{DEPLETING THE RELIC ABUNDANCE OF MILLICHARGED DM IN MATTER DOMINATED ERA}

In this section, we calculate the relic abundance of nonrelativistic millicharged DM, assuming that it gets decoupled during the modulus dominated era. As mentioned in Ref. [11], the annihilation cross section for a pair of millicharged particles is given by

$$
\langle\sigma v\rangle=\frac{\pi \alpha^{2} \epsilon^{2}}{m_{X}^{2}} k\left(\sqrt{1-\frac{m_{f}^{2}}{m_{X}^{2}}}\right)\left(1+\frac{m_{f}^{2}}{2 m_{X}^{2}}\right),
$$

where $k=1$ and $v^{2} / 6$ for the Dirac fermion and complex scalar, respectively; $\epsilon$ corresponds to charge; and $m_{f}$ corresponds to mass of fermion into which DM particles annihilate. We assume that DM is in the form of a Dirac fermion, so we will take $k=1$. Now, by using Eqs. (21) and (22), the relic abundance of millicharged DM particles will be parametrized by

$$
\Omega_{X}^{\prime \text { matter }} \approx 0.1\left[\frac{m_{\Phi}}{10^{3} \mathrm{TeV}}\right]^{5 / 4}\left[\frac{m_{X}}{20 \mathrm{MeV}}\right]^{3 / 2}\left[\frac{10^{-6}}{\epsilon}\right]^{2} \times\left(1-\frac{1}{52} \log \left[\left(\frac{20 \mathrm{MeV}}{m_{X}}\right)^{1 / 2}\left(\frac{\epsilon}{10^{-6}}\right)^{2}\left(\frac{10^{3} \mathrm{TeV}}{m_{\phi}}\right)^{1 / 4}\right]\right)^{3 / 2} .
$$


As discussed in Sec. II, millicharged DM particles are allowed to form only a fraction of the whole DM of the Universe. Thus, we parametrize the millicharged DM relic abundance by $\Omega_{X}^{\text {matter }}=f_{\mathrm{DM}} \Omega_{X}^{\text {matter }}$, where $f_{\mathrm{DM}}$ corresponds to the fraction of the total DM [10]. From Eq. (23), we can calculate the range of $\epsilon$ and $m_{X}$ for which the desired value of relic abundance $\left(\Omega_{X}^{\text {matter }}\right)$ can be obtained.

\section{A. Constraints on the mass of modulus}

Before we discuss the parameter space of mass and charge of millicharged DM obtained in the modulus dominated era, we will also discuss bounds on the modulus mass from both theoretical and experimental considerations. As discussed in Sec. III, the lower bound on the mass of the modulus $\left(m_{\Phi} \geq 10 \mathrm{TeV}\right)$ is already enforced from the requirement of not spoiling the predictions arising from BBN. On the other hand, in order to ensure that the DM gets decoupled in the matter dominated era, we have to keep $T_{\Gamma}<T_{\mathrm{FO}}$. Using Eq. (15), for $x_{\mathrm{FO}} \approx 10$ and $g_{* S}\left(T_{\Gamma}\right) \approx 100$, this requirement boils down to give

$$
m_{\Phi} \lesssim\left[10^{4}\left(\frac{m_{X}}{\mathrm{MeV}}\right)^{6 / 11}\right] \mathrm{TeV}
$$

Since we are interested in the mass range $1 \mathrm{MeV}<$ $m_{X}<1 \mathrm{GeV}$, the mass of modulus should always be less than $10^{4} \mathrm{TeV}$. Thus, we will have to satisfy the following bound on the mass of the modulus:

$$
10<\left(\frac{m_{\Phi}}{\mathrm{TeV}}\right)<10^{4} .
$$

\section{B. Allowed parameter space}

In Figs. 1(a)-(d), we show the parameter space of $\epsilon$ and $m_{X}$ in which $\Omega_{X}^{\text {matter }}$ forms a particular fraction $f_{\mathrm{DM}}$ of the whole DM of the Universe and also explain the observed $21-\mathrm{cm}$ signal reported by the EDGES Collaboration. We have shown results for $f_{\mathrm{DM}}=1,0.1,0.01,0.001$ as considered in Ref. [10]. It is clear from Eq. (23) that $\Omega_{X}^{\text {matter }}$ also depends on $m_{\Phi}$. We scan the parameter space by considering the aforementioned allowed range of $m_{\Phi}: 10 \mathrm{TeV}<m_{\Phi}<10^{4} \mathrm{TeV}$. It turns out that for $m_{\Phi} \leq$ $2.5 \times 10^{3} \mathrm{TeV}$ the value of $\epsilon$ will be less than the one allowed by the EDGES 21-cm signal, while for $m_{\Phi} \geq 6.5 \times 10^{3} \mathrm{TeV}$, it will be greater than the value of $\epsilon$ allowed by the EDGES $21-\mathrm{cm}$ signal. Therefore, we have fixed the value of the modulus mass $m_{\Phi}=2.5 \times 10^{3} \mathrm{TeV}$ in all the cases shown in Fig. 1. The black solid lines in Figs. 1(a)-(d) indicate the range of DM parameters required to explain the observed $21-\mathrm{cm}$ signal reported by the EDGES Collaboration. The dashed red lines indicate the parameter space in which millicharged DM abundance obtained by considering the matter dominated era corresponds to the quoted fraction $f_{\mathrm{DM}}$ of the whole DM of the Universe. We also compare our results with the parameter space allowed by DM freeze-out in the standard radiation dominated era. The black dashed lines in Figs. 1(a)-(d) indicate the parameter space required to get a particular fraction of millicharged DM if the same gets decoupled in the radiation dominated era. It can be easily figured out that dashed black lines lie quite above the solid black lines in the allowed range of parameter space, while the dashed red lines coincide with the solid black line for a particular point in the parameter space for all the cases presented in Fig. 1. From this, we can realize that the it is possible to have the desirable millicharged DM abundance in the allowed range of DM parameters required to explain the 21-cm line signal if the DM gets decoupled in the matter/modulus dominated era. In other words, we can deplete the overproduced relic abundance of millicharged DM (obtained by considering radiation) by considering the possibility of DM getting decoupled in the matter dominated era.

Now, the parameters of millicharged DM are also subjected to various experimental and astrophysical constraints. All the constraints are summarized in Sec. II. The shaded regions in Fig. 1 represent the regions ruled out by various experimental constraints. By taking these into account, we can realize that the region allowed to explain the $21-\mathrm{cm}$ absorption feature, along with the desirable relic abundance, is ruled out completely for the case of $f_{\mathrm{DM}}=1,0.1$ and 0.001 . For $f_{\mathrm{DM}}=0.01$, as we can see in Fig. 1, we get a point in the parameter space in which one can simultaneously explain the EDGES $21-\mathrm{cm}$ absorption feature and the right value of relic abundance while evading all other experimental constraints. This point corresponds to a particular value of modulus mass $m_{\Phi}=2.5 \times 10^{3} \mathrm{TeV}$.

We can see from Fig. 1(c) that the range of parameters free of all kinds of experimental constraints corresponds to $f_{\mathrm{DM}}=0.01,10 \mathrm{MeV} \lesssim m_{X} \lesssim 70 \mathrm{MeV}$ and $10^{-6} \lesssim$ $\epsilon \lesssim 10^{-5}$. In Fig. 2, we just show the results in this narrow range of $m_{X}$ by considering different values of the modulus mass. The black solid line shows the parameter space of the fraction of the millicharged $\mathrm{DM} f_{\mathrm{DM}}=0.01$ required to explain the $21-\mathrm{cm}$ line signal. The dashed red, dashed pink, dashed brown, dashed green, and dashed orange lines indicate the value of $\Omega_{X}^{\text {matter }} \sim 0.001$ for $m_{\Phi}=1.5 \times 10^{3} \mathrm{TeV}, \quad 2.5 \times 10^{3} \mathrm{TeV}, \quad 4.5 \times 10^{3} \mathrm{TeV}$, $6.5 \times 10^{3} \mathrm{TeV}$, and $7.5 \times 10^{3} \mathrm{TeV}$, respectively. We can clearly see that for $m_{\Phi} \leq 2.5 \times 10^{3} \mathrm{TeV}$ and $m_{\Phi} \geq$ $6.5 \times 10^{3} \mathrm{TeV}$ the lines representing the parameter space of $f_{\mathrm{DM}}=0.01$ lie below and above the black solid line, respectively, for the entire range of $m_{X}$ shown in Fig. 2. Thus, the region allowed by the $21-\mathrm{cm}$ line signal basically sets a constraint on the modulus mass $m_{\Phi} \approx(2.5-6.5) \times 10^{3} \mathrm{TeV}$. 


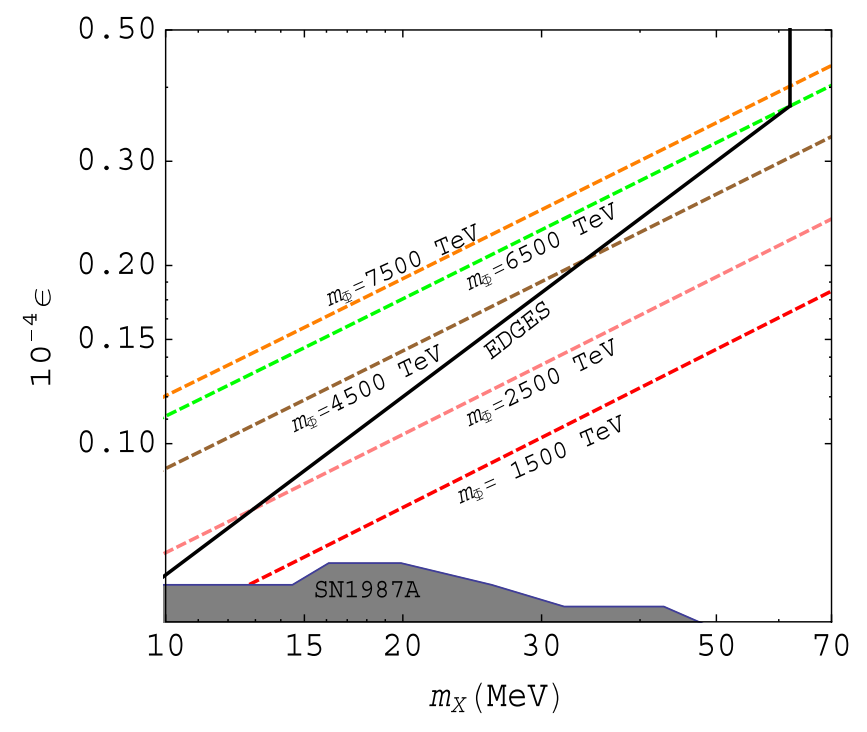

FIG. 2. Here, we show our results of DM relic abundance in the narrow range allowed by the EDGES $21-\mathrm{cm}$ absorption feature. The black solid line shows the parameter space of the fraction of the millicharged DM $f_{\mathrm{DM}}=0.01$ required to explain the $21-\mathrm{cm}$ line signal. The dashed red, dashed pink, dashed brown, dashed green, and dashed orange lines indicate the value of $\Omega_{X}^{\text {matter }} \sim$ 0.001 (i.e., $f_{\mathrm{DM}}=0.01$ ) for $m_{\Phi}=1.5 \times 10^{3} \mathrm{TeV}, 2.5 \times 10^{3} \mathrm{TeV}$, $4.5 \times 10^{3} \mathrm{TeV}, 6.5 \times 10^{3} \mathrm{TeV}$, and $7.5 \times 10^{3} \mathrm{TeV}$, respectively. We can see that for $m_{\Phi}=1.5 \times 10^{3} \mathrm{TeV}$ and $7.5 \times 10^{3} \mathrm{TeV}$ the lines representing the parameter space of $f_{\mathrm{DM}}=0.01$ lie below and above the black solid line, respectively, for the entire range of $m_{X}$ allowed by the $21-\mathrm{cm}$ signal. Thus, the region allowed by the 21-cm line signal sets a constraint on the modulus mass $m_{\Phi} \approx(2.5-6.5) \times 10^{3} \mathrm{TeV}$.

With this, we show that the overproduction of DM in the requisite range of mass and charge of DM mentioned in Ref. [11] can be evaded if we take into account the presence of the modulus dominated era in the early Universe.

\section{CONCLUSIONS}

Previous studies based on the millicharged DM explanation of the EDGES $21-\mathrm{cm}$ absorption signal argue that the energy density of millicharged DM $\left(f_{\mathrm{DM}} \Omega_{X}\right)$ obtained by considering thermal annihilation through massless photons will overclose the Universe for the range of parameters allowed by the $21-\mathrm{cm}$ line signal $[10,11]$. The overproduced relic density can be depleted by considering extra force carriers, such as the $U(1)_{L_{\mu}-L_{\tau}}$ gauge group. However, the yet unconstrained tight parameter space allowed in this case is expected to be within the reach of future measurements $[11,13]$. Thus, one needs a mechanism to deplete the overproduced relic abundance of millicharged DM. In this article, we have investigated the possibility of depleting the energy density of millicharged DM particles by taking into account the existence of an early moduli dominated era. Moduli are a ubiquitous prediction resulting from the consistent string compactifications [20]. Since the successful UV completion of any inflationary model requires its embedding in string theory, we are compelled to consider the presence of moduli in the early Universe. The dynamics of moduli tend to change the standard thermal history of the Universe, leading to an early matter dominated era. Interestingly, they also allow for a richer range of possibilities for the production of DM in the early Universe. Based on the values of mass and charged allowed by the $21-\mathrm{cm}$ line signal, we realize that the millicharged DM might get decoupled in an early modulus dominated era in our case. Thus, we have calculated the relic abundance of millicharged DM particles for this case. The energy density of DM particles obtained in this case is expected to be less than the one obtained in the standard radiation dominated case because i) the expansion rate of the Universe is different for the matter and radiation dominated eras and ii) a significant fraction of the energy density gets diluted because of the decay of the modulus. We found that for the value of modulus mass around $m_{\Phi} \approx(2.5-6.5) \times 10^{3} \mathrm{TeV}$ the millicharged DM particles could actually yield the desired fraction of DM particles in the region allowed by the $21-\mathrm{cm}$ line signal reported by the EDGES Collaboration. Thus, the overproduction of the relic density can be circumvented in case of an early modulus dominated era. We have discussed the case of the millicharged DM interacting with baryons through SM photons. However, it can be extended easily for a case with a light massive mediator.

Furthermore, quite interestingly, an almost similar value of $m_{\Phi}$ is required to obtain the observed bounds on the inflationary predictions and $\mathcal{O}(\mathrm{TeV})$-scale supersymmetry in one of the promising string compactification scenarios, dubbed the large volume scenario [24-27]. Thus, the results from $21-\mathrm{cm}$ experiments might provide a new window into the dark sector, which might give hints of the nongravitational interaction of $\mathrm{DM}$ and $\mathcal{O}(\mathrm{TeV})$-scale supersymmetry in the future.

\section{ACKNOWLEDGMENTS}

The work of M. D. is supported by Department of Science and Technology, Government of India, under Grant No. IFA18-PH215 (INSPIRE Faculty Award). M. D. would like to thank Julion B. Munoz (Harvard University) for useful comments on an earlier draft of the manuscript. M. D. would like to acknowledge Vikram Rentala (IIT Bombay, India) for several useful discussions on the EDGES results and Gaurav Goswami (Ahmedabad University, India) for some useful clarifications. 
[1] M. Mateo, Annu. Rev. Astron. Astrophys. 36, 435 (1998).

[2] D. N. Spergel and P. J. Steinhardt, Phys. Rev. Lett. 84, 3760 (2000).

[3] J. D. Bowman, A. E. E. Rogers, R. A. Monsalve, T. J. Mozdzen, and N. Mahesh, Nature (London) 555, 67 (2018).

[4] R. Barkana, Nature (London) 555, 71 (2018).

[5] A. Fialkov, R. Barkana, and A. Cohen, Phys. Rev. Lett. 121, 011101 (2018).

[6] J. B. Muoz, C. Dvorkin, and A. Loeb, Phys. Rev. Lett. 121, 121301 (2018).

[7] H. Tashiro, K. Kadota, and J. Silk, Phys. Rev. D 90, 083522 (2014).

[8] J. B. Muoz, E. D. Kovetz, and Y. Ali-Hamoud, Phys. Rev. D 92, 083528 (2015).

[9] R. Barkana, N. J. Outmezguine, D. Redigolo, and T. Volansky, Phys. Rev. D 98, 103005 (2018).

[10] J. B. Muoz and A. Loeb, Nature (London) 557, 684 (2018).

[11] A. Berlin, D. Hooper, G. Krnjaic, and S. D. McDermott, Phys. Rev. Lett. 121, 011102 (2018).

[12] E. D. Kovetz, V. Poulin, V. Gluscevic, K. K. Boddy, R. Barkana, and M. Kamionkowski, Phys. Rev. D 98, 103529 (2018).

[13] N. Klop and S. Ando, Phys. Rev. D 98, 103004 (2018).

[14] E. D. Kovetz, I. Cholis, and D. E. Kaplan, Phys. Rev. D 99, 123511 (2019).

[15] G. D. Coughlan, W. Fischler, E. W. Kolb, S. Raby, and G. G. Ross, Phys. Lett. 131B, 59 (1983).

[16] T. Banks, D. B. Kaplan, and A. E. Nelson, Phys. Rev. D 49, 779 (1994).

[17] B. de Carlos, J. A. Casas, F. Quevedo, and E. Roulet, Phys. Lett. B 318, 447 (1993).

[18] M. Dine, Phys. Lett. B 482, 213 (2000).

[19] B. S. Acharya, P. Kumar, K. Bobkov, G. Kane, J. Shao, and S. Watson, J. High Energy Phys. 06 (2008) 064.
[20] G. Kane, K. Sinha, and S. Watson, Int. J. Mod. Phys. D 24, 1530022 (2015).

[21] R. Allahverdi, B. Dutta, and K. Sinha, Phys. Rev. D 83, 083502 (2011).

[22] R. Allahverdi, M. Cicoli, B. Dutta, and K. Sinha, Phys. Rev. D 88, 095015 (2013).

[23] A. L. Erickcek, Phys. Rev. D 92, 103505 (2015).

[24] V. Balasubramanian, P. Berglund, J. P. Conlon, and F. Quevedo, J. High Energy Phys. 03 (2005) 007.

[25] R. Blumenhagen, J. P. Conlon, S. Krippendorf, S. Moster, and F. Quevedo, J. High Energy Phys. 09 (2009) 007.

[26] M. Cicoli, J. P. Conlon, and F. Quevedo, Phys. Rev. D 87, 043520 (2013).

[27] L. Aparicio, M. Cicoli, S. Krippendorf, A. Maharana, F. Muia, and F. Quevedo, J. High Energy Phys. 11 (2014) 071.

[28] The requirement of modulus mass around $10^{3} \mathrm{TeV}$ for TeVscale supersymmetry is explicitly mentioned at the end of Sec. II of Ref. [26].

[29] J. H. Chang, R. Essig, and S. D. McDermott, J. High Energy Phys. 09 (2018) 051.

[30] A. A. Prinz et al., Phys. Rev. Lett. 81, 1175 (1998).

[31] C. Boehm, M. J. Dolan, and C. McCabe, J. Cosmol. Astropart. Phys. 08 (2013) 041.

[32] P. A. R. Ade et al. (Planck Collaboration), Astron. Astrophys. 594, A13 (2016).

[33] W. L. Xu, C. Dvorkin, and A. Chael, Phys. Rev. D 97, 103530 (2018).

[34] In our case, the constraints on $\Delta N_{\text {eff }}$ as given in Ref. [31] can be applied only if the modulus reheating temperature is greater than the BBN temperature.

[35] In this paper, we will take into account the presence of a single massless field; thus, we will always refer to the "modulus" in forthcoming sections.

[36] S. Hamdan and J. Unwin, Mod. Phys. Lett. A 33, 1850181 (2018). 